\title{
Instrumentos de avaliação da ansiedade da criança hospitalizada
}

Anxiety assessment tools in hospitalized children

Instrumentos de evaluación de ansiedad de niños hospitalizados

Fernanda Vieira de Campos ${ }^{1}$ io https://orcid.org/0000-0003-0315-995X

Camila Forni Antunes' ${ }^{1}$ ib https://orcid. org/0000-0003-2981-262X

Elaine Buchhorn Cintra Damião1 io https://orcid.org/0000-0001-7435-3130

Lisabelle Mariano Rossato ${ }^{1}$ io https://orcid.org/0000-0003-3375-8101

Lucila Castanheira Nascimento ${ }^{1}$ id https://orcid.org/0000-0002-7900-7111

\section{Como citar:}

Campos FV, Antunes CF, Damião EB, Rossato

LM, Nascimento LC. Instrumentos de avaliação

da ansiedade da criança hospitalizada. Acta Paul Enferm. 2020;33:eAPE20180250.

DOI

http://dx.doi.org/10.37689/actaape/2020AR02505

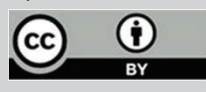

Descritores

Criança; Criança hospitalizada; Ansiedade: Escala de ansiedade frente a teste; Enfermagem pediátrica

Keywords

Child; Child, hospitalized; Anxiety: Test anxiety scale; Pediatric nursing

Descriptores

Niño; Niño hospitalizado; Ansiedad; Escala de ansiedad ante pruebas; Enfermería pediátrica

Submetido 7 de Outubro de 2018

Aceito

23 de Março de 2020

Autor correspondente Elaine Buchhorn Cintra Damião E-mail: buchhorn@usp.br

\section{Resumo}

Objetivo: Identificar na literatura os instrumentos existentes para a avaliação da ansiedade em crianças hospitalizadas.

Métodos: Trata-se de uma revisão integrativa. As buscas foram realizadas de janeiro a maio de 2017 nas seguintes bases de dados: PubMed, LILACS e CINAHL, com os descritores anxiety, hospitalized child, inpatients, children, emotional responses, emotional manifestation, emotional reactions, validation studies, manifest anxiety scale e test anxiety scale.

Resultados: Após a seleção, foram analisados 33 artigos, sendo identificados dez instrumentos para a avaliação da ansiedade da criança. Oito instrumentos utilizaram a Escala de Likert nas respostas, um empregou a escala analógica de um a dez e 0 único instrumento que utilizou uma estratégia lúdica, o desenho dirigido, foi o Child Drawing: Hospital.

Conclusão: Recomenda-se a tradução, adaptação transcultural e validação do instrumento CD:H. Este estudo visa contribuir para 0 conhecimento dos enfermeiros pediatras no manejo da ansiedade da criança em unidades pediátricas, a fim de diminuir seu sofrimento emocional durante a hospitalização.

\section{Abstract}

Objective: Identify existing tools for the assessment of anxiety in hospitalized children in the literature.

Methods: An integrative review was undertaken. The searches took place from January to May 2017 in the following databases: PubMed, LILACS and CINAHL, using the descriptors anxiety, hospitalized child, inpatients, children, emotional responses, emotional manifestation, emotional reactions, validation studies, manifest anxiety scale and test anxiety scale.

Results: After the selection, 33 articles were analyzed, in which 10 child anxiety assessment tools were identified. Eight instruments used the Likert scale in the answers, one used the analogue scale from one to ten and the only instrument that used a playful strategy, directed drawing, was the Child Drawing: Hospital.

Conclusion: The translation, cross-cultural adaptation and validation of the $\mathrm{CD}: \mathrm{H}$ is recommended. This study aims to contribute to pediatric nurses' knowledge in the management of child anxiety in pediatric wards, with a view to reducing their emotional suffering during hospitalization.

\section{Resumen}

Objetivo: Identificar en la literatura los instrumentos existentes para evaluar la ansiedad de niños hospitalizados. Métodos: Se trata de una revisión integradora. Las búsquedas se realizaron de enero a mayo de 2017 en las 
siguientes bases de datos: PubMed, LILACS y CINAHL, con los descriptores anxiety, hospitalized child, inpatients, children, emotional responses, emotional manifestation, emotional reactions, validation studies, manifest anxiety scale y test anxiety scale.

Resultados: Luego de realizar la selección, se analizaron 33 artículos, en los que se identificaron diez instrumentos para evaluar la ansiedad de niños. Ocho instrumentos utilizaron la escala de Likert en las respuestas, uno empleó la escala analógica de uno a diez y el único instrumento que utilizó una estrategia lúdica, el dibujo dirigido, fue el Child Drawing: Hospital.

Conclusión: Se recomienda la traducción, adaptación transcultural y validación del instrumento CH:H. Este estudio tiene el objetivo de contribuir para el conocimiento de los enfermeros pediatras en el manejo de la ansiedad de los niños en unidades pediátricas, a fin de reducir su sufrimiento emocional durante la hospitalización.

\section{Introdução}

O crescente avanço da Medicina e a melhoria dos processos científicos de diagnóstico e terapia elevaram a sobrevida de crianças criticamente doentes e das que possuem doença crônica. As internaçóes tornaram-se prolongadas, ocasionando o afastamento de casa e do ambiente escolar por grandes períodos, aumentando o sofrimento, dor e angústia. ${ }^{(1)}$

Estudos têm descrito as percepçóes das crianças escolares hospitalizadas, além de demonstrar a importância em incluí-las em seu processo de cuidado, valorizando seus desejos e sua singularidade por meio da comunicação, do lúdico e de brinquedos durante os atendimentos, a fim de minimizar o medo e a ansiedade durante a hospitalização. ${ }^{(2-4)}$

A ansiedade pode ser definida como um "sentimento de apreensão causado por antecipaçáo de perigo, interno ou externo", possuindo características fisiológicas e psicológicas, como medo, insegurança, tensão, dor muscular, tremores, sudorese, taquicardia e taquipneia. A ansiedade surge geralmente em situaçôes de sensação de ameaça e perigo iminente, porém inespecífico. ${ }^{(5)}$

A ansiedade, por ser mais abrangente, abstrata e difícil de ser definida pelas crianças, torna mais difícil sua identificação, sendo necessária a aplicação de instrumentos e estratégias específicas de avaliaçáo dos sinais e sintomas apresentados pela criança. ${ }^{(6,7)}$

Nesse sentido, objetivou-se identificar na literatura os instrumentos existentes para a avaliação da ansiedade em crianças hospitalizadas, com o intuito de instrumentalizar os enfermeiros pediatras a identificarem os sintomas de ansiedade da criança e assim proporem estratégias e intervençôes adequadas às situações vivenciadas pela criança no hospital.

\section{Métodos}

O estudo é uma revisão integrativa da literatura. Foram seguidas as subsequentes etapas:

a. Seleção da pergunta de estudo: Qual é o conhecimento atual sobre a avaliaçáo da ansiedade da criança em processo de hospitalização;

b. Estabelecimento de critérios para seleçáo da amostra: artigos sobre instrumentos de avaliação da ansiedade de crianças, publicadas nos últimos 10 anos (2007-2017), nas línguas inglesa, espanhola e portuguesa, nas bases de dados PubMed, LILACS e CINAHL;

c. Busca na literatura: utilização dos descritores adequados à pergunta de pesquisa em várias combinações nas bases de dados mencionadas anteriormente. Busca ativa ou manual dos artigos por meio dos artigos similares indicados pelas bases de dados;

d. Definição de informaçóes a serem extraídas dos estudos a serem selecionados: Título do artigo e autores; periódico, número, volume e ano de publicação; país; escala utilizada; metodologia; resultados;

e. Avaliação dos estudos incluídos, interpretação de resultados e apresentação da revisão: análise das várias escalas encontradas, segundo sua validade e aplicabilidade em nosso contexto. ${ }^{(8)}$ A questão norteadora do estudo foi "Quais são os instrumentos para avaliaçáo da ansiedade em crianças descritos na literatura científica?". Utilizouse, ainda, a estratégia $\mathrm{PICO},{ }^{(9)}$ onde:

- $\mathrm{P}($ paciente $)=$ crianças; crianças hospitalizadas

- I (Intervenção) $=$ instrumentos de avaliaçáo da ansiedade;

- $\mathrm{C}$ (comparação) $=$ não foi utilizada;

- $\mathrm{O}$ (outcomes - desfecho) = identificação dos sintomas de ansiedade na criança. 


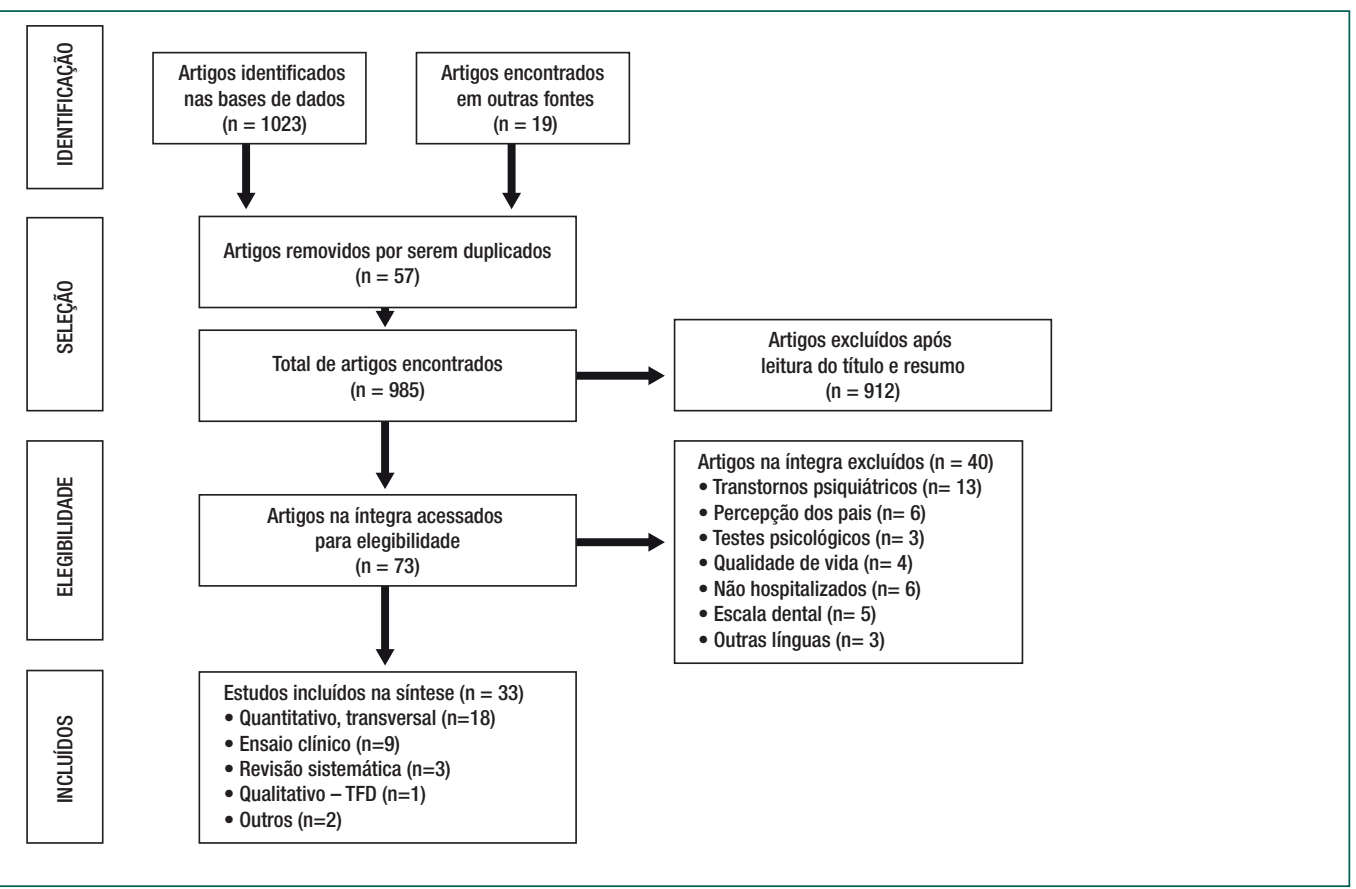

Figura 1. Fluxo da informação com as diferentes fases de uma revisão sistemática

Desse modo, foi possível identificar os descritores para a busca nas bases eletrônicas PubMed, as LILACS e CINAHL, nos últimos dez anos, realizada no período de janeiro a maio de 2017, utilizando-se os seguintes, descritores: anxiety, hospitalized child, inpatients, children, emotional responses, emotional manifestation, emotional reactions, validation studies, manifest anxiety scale e test anxiety scale. Em cada base de dados os descritores eram combinados de forma variada; inpatient $x$ validation studies $x$ child; inpatient $x$ validation studies $x$ anxiety; inpatient $x$ anxiety $x$ child; hospitalized child $x$ validation studies; manifest anxiety scale $x$ child; entre outras combinaçóes. O mesmo foi realizado nas bases de dados LILACS e CINAHL. No total obteve-se 1023 artigos, após a exclusão de artigos em outros idiomas e outros temas, como: aspectos clínicos, transtornos psíquicos, nutrição, dados administrativos dentre outros. Além disso, realizou-se uma busca ativa e manual a partir dos 'artigos similares' aos artigos encontrados, segundo indicado por cada base de dados, tendo sido encontrados mais 19 artigos, totalizando 1042 artigos.

Em seguida, 57 artigos foram removidos por serem duplicados e 912 foram excluídos após leitura do título, restando 73 artigos. Após leitura do resumo e textos na íntegra, foram excluídos 40 artigos, totalizando, ao final, 33 artigos, os quais foram analisados. Os critérios de exclusão adotados foram artigos não disponíveis em inglês, português ou espanhol $(n=3)$, artigos os quais abordavam qualidade de vida $(n=4)$, transtornos psiquiátricos $(n=13)$, testes psicológicos $(n=3)$, crianças não hospitalizadas $(n=6)$, escalas dentais $(n=5)$ e relatos e escalas preenchidas sob a ótica dos pais $(n=6)$. Ao final, foram selecionados 33 artigos com as principais escalas e instrumentos de avaliação utilizados para analisar e mensurar a ansiedade da criança hospitalizada. Entre esses artigos, foram encontrados estudos de validação, revisões sistemáticas e integrativas e ensaios clínicos randomizados, utilizando as dez escalas selecionadas. A figura 1 apresenta o fluxograma conforme recomendação PRISMA. ${ }^{(10)}$

\section{Resultados}

As revisóes integrativas além de trazer o estado da arte sobre determinado tema, também contribui para que o profissional enfermeiro tenha acesso de maneira simples e atualizada sobre tópicos de seu interesse, a fim de embasar cientificamente o cuidado prestado à criança. Foram identificados dez 
instrumentos, que avaliam a ansiedade das crianças, que apresentamos a seguir.

\section{Revised children's manifest anxiety scale - RCMAS - Escala de ansiedade infantil - "O que penso e sinto"}

A RCMAS é a revisão da Children's Manifest Scale - CMAS, de 1956, ${ }^{(1)}$ que inicialmente possuía 53 itens. Foi readaptada e validada por Reynolds e Richmond $^{(12,13)}$ e Richmond e Millar, ${ }^{(14)}$ para testes psicológicos em crianças e adolescentes de 6 a 19 anos. É conhecida como "O que eu penso e sinto", possui 37 itens de autorrelato para avaliação da ansiedade de crianças e adolescentes com idade entre 7 e 17 anos. Pode ser aplicada individualmente ou em grupo, entretanto foi desenvolvida para ser respondida pela própria pessoa. É dividida em escala de ansiedade e escala da mentira.

A escala da ansiedade tem pontuação total calculada sobre 28 itens divididos em três fatores: Fisiológico, Preocupação e Concentração. ${ }^{(12-14)}$

Os nove itens restantes constituem o domínio 'Escala da Mentira', que é uma avaliação de quanto a criança necessita da aprovação dos pais e/ou dos pares. A escala possui dois fatores e as respostas podem ser "sim" ou "não". Pontuaçôes altas nas subescalas representam diferentes tipos e origens de ansiedade. ${ }^{(12-14)}$

A escala foi traduzida e adaptada para o português, porém não foi validada com crianças escolares hospitalizadas. ${ }^{(15)} \mathrm{Na}$ escala brasileira, houve uma pequena modificação na divisão dos fatores: sete itens para o Fisiológico, dez itens para o fator Preocupação e 11 itens em Concentração, mantendo os dois fatores da escala da mentira, totalizando 37 itens. A validação foi considerada bem-sucedida, com as qualidades psicométricas com resultados bastante semelhantes aos de estudos internacionais. ${ }^{(15)}$

\section{Screen for child anxiety related emotional disorders - child version. (SCARED - C)}

Objetivou-se criar um instrumento que fosse confiável para ser utilizado no rastreamento de desordens de ansiedade de crianças e adolescentes: Transtorno de ansiedade geral, Distúrbio da Ansiedade da Separação, Síndrome do Pânico, Fobia Social e
Fobia da Escola. Criado inicialmente com 85 itens, foi reduzido a 38 e, finalmente, validado com 41 itens. Em todas as versóes, foram mantidos cinco fatores: somático/pânico - 13 itens; ansiedade generalizada - nove itens; ansiedade de separação - oito itens; fobia social - sete itens e fobia escolar - quatro itens. A pontuação de cada item varia em uma escala de Likert de zero a dois. Para cada item, a criança escolhe a resposta que melhor descreve como ela tem se sentido nos últimos três meses, com pontuação total de zero a 82, sendo que altas pontuaçóes revelam elevados níveis de ansiedade. ${ }^{(16,17)}$

Foi validada para a língua portuguesa falada no Brasil por Isolan et al., ${ }^{(18)}$ sendo mantidos os mesmos fatores da escala original. A escala é de fácil e rápida administração, bem como de baixo custo. ${ }^{(18)}$

\section{Children's depression inventory - CDI - Inventário de Depressão Infantil)}

Utilizado majoritariamente para fornecer dados de transtornos psiquiátricos, mais especificamente, a depressão. Porém também é utilizado em ensaios clínicos randomizados para mensurar níveis de ansiedade. ${ }^{(19)}$ Desenvolvido por Kovacs ${ }^{(20)}$ para crianças e adolescentes de 7 a 17 anos, o inventário é uma adaptação do Beck Depression Inventory - BDI, ${ }^{(21)}$ é composto por 27 questóes alternativas de autorrelato ou respondidas por outros informantes, tais como familiares ou professores. A escala é dividida em cinco fatores, entre sintomas afetivos, cognitivos e sociais: humor negativo, problemas interpessoais, ineficácia, anedonia e autoestima negativa. Os itens referem-se aos sentimentos percebidos pela criança nas duas últimas semanas, sendo pontuação zero quando a primeira alternativa é escolhida (ausência de sintoma); um quando a segunda alternativa é escolhida (sintoma leve) e dois quando a terceira alternativa é escolhida (sintoma claro) e a sua pontuação total variável de zero a 54. Traços de depressão são considerados positivos quando o escore é igual ou maior que 18. Existem variaçóes da escala validadas em diversas versôes, entre elas, resumidas e em outras línguas. Este questionário foi adaptado no Brasil por Gouveia et al. ${ }^{(22)}$ e posteriormente validado por Golfeto et al., ${ }^{(23)}$ já com 20 itens e ponto de corte 17 , ou seja, escores acima de 17 indicam pro- 
váveis quadros depressivos. Cruvinel, Boruchovitch e Santos, ${ }^{(24)}$ em sua validação, não chegaram à conclusão de quantos itens são necessários ao inventário, uma vez que utilizaram o estudo original de Kovacs $^{(20)}$ e o adaptado por Gouveia et al. ${ }^{(22)} \mathrm{em}$ amostras separadas e com resultados diferentes.

\section{Multidimensional anxiety scale for children - MASC - Escala Multidimensional de Ansiedade para Crianças - MASC - VB}

Esta é uma escala de autorrelato para avaliar a ansiedade de crianças e adolescentes com idade entre oito e 19 anos nos domínios afetivo, físico, cognitivo e comportamental. O instrumento pode ser aplicado individualmente ou em grupo, porém deve ser respondido pela própria pessoa. Composta por 39 itens distribuídos em quatro fatores: sintomas físicos (tensão / inquietude; somáticos / autonômicos), ansiedade social (humilhação e medo de rejeição pública), prevenção de danos (enfrentamentos e perfeccionismo) e ansiedade de separação. As respostas têm quatro opçóes de pontuação na escala Likert. ${ }^{(25-27)}$ Foi validada e adaptada culturalmente no Brasil como MASC VB, por Nunes ${ }^{(28)}$ em sua dissertação de mestrado e utilizada por Vianna ${ }^{(25)}$ em sua pesquisa de mestrado. Ambos os autores referem que a escala possui bom desempenho psicométrico para ser utilizada na população brasileira. ${ }^{(25,28)}$

\section{State trait inventory for children - STAI-C - Inventário de Ansiedade Traço Estado para criança - IDATE - C}

A escala IDATE-C objetiva avaliar dois tipos de ansiedade na criança e adolescentes com idade entre 9 e 12 anos: a 'ansiedade-traço', que se refere às diferenças individuais que favorecem a ansiedade da pessoa e a 'ansiedade-estado', que se relaciona à diferença na tendência de se reagir com aumento de intensidade à ansiedade do momento. ${ }^{(29,30)}$

É constituída por duas escalas de autorrelato com 20 itens cada, com três opçóes de respostas e escores total de 20 a 60 em cada uma. ${ }^{(29)}$ É perguntado à criança como ela geralmente se sente (para mensurar ansiedade-traço) e como está se sentindo naquele momento (para mensurar ansiedade-esta- do), com quatro opçóes de respostas cada um. A ansiedade-estado é caracterizada como um estado emocional transitório, em que os escores podem variar em intensidade e aumentar ou diminuir com o passar do tempo; a ansiedade-traço é caracterizada como uma resposta ansiosa permanente da personalidade do indivíduo. Validada no Brasil como IDATE: Inventário de Ansiedade Traço-Estado. ${ }^{(30)}$

\section{State anxiety scale for children - SASC-R}

Criada para determinar primariamente o nível de ansiedade social das crianças e suas relaçóes sociais por La Greca et al. ${ }^{(31)}$ em 1988 e posteriormente revisada por La Greca e Stone em $1993^{(32)}$. Atualmente também é utilizada para determinar o nível de ansiedade de crianças submetidas a procedimentos dolorosos e cirurgias. Composta por 26 itens, divididos em três fatores: medo da avaliação negativa dos colegas, prevenção social e angústia específica para novas situaçôes e prevenção e angústia social generalizada. Cada item tem uma pontuação de um a cinco. ${ }^{(31,32)}$ Traduzida em diversas línguas, porém ainda não em português.

\section{Children's emotional manifestation scale - CEMS}

Escala observacional desenvolvida e validada por $\mathrm{Li}$ e Lopez ${ }^{(33)}$ para analisar a ansiedade em crianças no pré-operatório ou antes de algum procedimento invasivo, estressante ou doloroso. É composta por cinco categorias comportamentais; cada categoria consiste em observar cinco comportamentos: expressão facial, vocalização, atividade, interação e nível de cooperação, sendo que cada uma dessas categorias possui um escore de um a cinco, dependendo do nível e intensidade. $\mathrm{O}$ total da pontuação pode variar de cinco a 25. Altos escores indicam altos comportamentos de ansiedade e emoçôes negativas. ${ }^{(33)}$ Essa escala ainda não foi traduzida para o português.

\section{Emotional reaction instrument checklist (ERC)}

Desenvolvido para mensurar as respostas emocionais positivas e negativas das crianças de sete a 18 anos quanto à hospitalização e cirurgias. Possui 16 itens de autorrelato que têm até quatro pontos de cada item, em uma escala de zero a três, sendo um - de modo nenhum a quatro - realmente, divididos em sete itens 
emocionais: feliz, triste, exaltado, bravo, calmo/relaxado, com medo/assustado e nervoso/preocupado, e as crianças devem responder aos números e não às sentenças. Altos escores na escala indicam maiores emoçôes positivas ou menos angústias. ${ }^{(34-36)} \mathrm{O}$ instrumento também foi validado em coreano como ERI-K ${ }^{(35)}$ e inglês como ERI-E. ${ }^{(36)}$ Escala não traduzida para o português.

\section{Escala visual analógica - VAS}

Consiste em um traço de linha horizontal de $10 \mathrm{~cm}$, com diferentes termos e frases como: "Não estou ansioso" e no final "eu estou muito ansioso", em que é solicitado à criança responder ou marcar aquele que lhe é mais conveniente, sendo zero o mínimo de ansiedade e dez o máximo. A aplicação da escala implica que a criança saiba ler e tenha o desenvolvimento cognitivo suficiente para compreender o significado da escala e saber se autoavaliar. Apesar da limitação, os autores reforçam o caráter simples e rápido da escala, podendo ser utilizada em variados cenários. $^{(37)} \mathrm{A}$ escala analógica é mais comumente utilizada na mensuração de dor. ${ }^{(38,39)}$

\section{Child drawing hospital - CD:H}

O instrumento Child Drawing: Hospital, criado pelas enfermeiras Clatworthy, Simon e Tiedeman, ${ }^{(40,41)}$ foi designado para mensurar a ansiedade de crianças em idade escolar hospitalizadas a partir de seu próprio ponto de vista e desperta interesse devido sua fácil aplicabilidade para a prática clínica hospitalar. No momento da aplicação o paciente é convidado a realizar um desenho de uma pessoa no hospital. A partir do desenho, o enfermeiro pediatra poderá avaliá-lo por meio do instrumento do $\mathrm{CD}: \mathrm{H}$, pontuando as características desenhadas e obtendo o nível de ansiedade em que a criança se encontra. $\mathrm{O}$ CD:H consiste em um manual com orientaçóes para a avaliação do desenho e um gabarito com os escores relacionados aos itens avaliados. ${ }^{(40)}$

O desenho produzido receberá pontos de acordo com a classificação do instrumento do $\mathrm{CD}: \mathrm{H}$. O $\mathrm{CD}: \mathrm{H}$ é divido em três partes: A, B e C. A Parte A é constituída de 14 itens que avaliam: posição; ação; comprimento; largura; tamanho da pessoa; expressão facial e olhos; cor predominante; número de cores utilizadas; uso do papel; localização no papel; inclusão e tamanho de equipamentos hospitalares e nível de desenvolvimento. A parte B é composta por oito itens que irão presumir índices altos de ansiedade, como por exemplo: se há ou não omissão ou exagero de alguma parte do corpo desenhado pela criança. A parte $\mathrm{C}$ refere-se sobre o gestalt do desenho, ou seja, o avaliador irá pontuar o efeito que o desenho produz em sua pessoa, variando de agradável a oprimido. ${ }^{(40)}$

\section{Discussão}

A adaptação da criança à internação hospitalar depende de fatores exógenos e endógenos, sendo o primeiro dependente das atitudes dos pais e o tempo de permanência no hospital, adicionado às experiências vivenciadas anteriormente. Já os fatores endógenos são as características pessoais de cada criança para enfrentar a doença. Sendo assim, devido aos mecanismos de adaptação e percepção da criança não estarem totalmente desenvolvidos, o enfrentamento à hospitalização pode desencadear sentimentos estressantes, como a ansiedade. ${ }^{(42)}$

Dentre as necessidades de cuidado na hospitalização da criança, além dos aspectos físicos, estão a avaliação, acompanhamento e tratamento dos aspectos emocionais e sociais, a fim de minimizar o sofrimento. Adicionado a isso, vale ressaltar que o processo de hospitalização não é enfrentado igualmente por todas as crianças. ${ }^{(43)}$

Nesse sentido, é importante que o enfermeiro utilize instrumentos validados em nosso idioma a fim de identificar precocemente os sintomas do comportamento de ansiedade.

$\mathrm{O}$ instrumento Child Drawing: Hospital foi o único instrumento encontrado que permite que as crianças sejam avaliadas por um estratégia lúdica como o desenho. O desenho infantil é um instrumento que permite a criança expressar seus sentimentos e angústias, uma vez que ainda não possui a capacidade de verbalizar seu estado emocional. É por meio do desenho que a criança organiza, processa informações e desenvolve os conceitos. ${ }^{(4)}$ Além disso, desenhar é uma atividade agradável, sendo uma tarefa comum em sua experiência de vida. 
Conforme a criança cresce, o desenho da figura humana começa a emergir. Por volta dos seis anos começam a aparecer os desenhos com cabeça e tronco e aos sete anos surgem traços mais precisos com a inclusão de outras partes do corpo como os joelhos e calcanhares. ${ }^{(44,45)}$ Em torno dos nove anos até o início da adolescência os desenhos são realistas, contendo maior quantidade de detalhes e diferenciação sexual. Além disso, as crianças tornam-se mais críticas com seu desenho para que seja o mais real possível. ${ }^{(46,47)}$

O uso projetivo de desenhos permite que o profissional obtenha uma amostra inicial do modo como o indivíduo se comporta ou reage diante de certas situaçóes consideravelmente não estruturadas, além de permitir uma maior interação entre o profissional e o paciente. ${ }^{(48,49)}$ Para Freitas ${ }^{(43)}$ o desenho solicitado à criança hospitalizada possibilita melhor obtenção de dados no que diz respeito à compreensão de seu quadro clínico, bem como das emoçóes que emergem deste. Além disso, apesar de ser apenas uma etapa do processo de avaliação, ele permite que a criança organize informaçóes, processe experiências vividas, sendo estimulada a desenvolver sua representação singular do mundo. ${ }^{(50,51)}$

Vale ressaltar que a técnica projetiva de desenho não oferece um diagnóstico do estado emocional da criança como todo, mas fornece informaçóes sobre como a criança está se sentindo naquele momento. Caso seja necessário, principalmente para os mais ansiosos, a criança deverá ser encaminhada a profissionais especializados, como psicólogos e psiquiatras, a fim de realizar avaliaçóes mais aprofundadas. ${ }^{(47)}$

É imprescindível que o enfermeiro reconheça os sintomas da ansiedade por meio das reaçóes do escolar, de modo a estar alerta aos indícios físicos, emocionais e comportamentais. ${ }^{(7)}$ Além disso, os enfermeiros devem proporcionar momentos que reduzam sentimentos de ansiedade, utilizando técnicas com brinquedos, histórias e desenhos, sendo estas, medidas projetivas para avaliar estados emocionais da infância. Portanto, o desenho da figura humana garante a identificação precoce de problemas psicológicos e uma melhor compreensão do estado emocional ao qual a criança se encontra no momento. ${ }^{(47,50)}$

\section{Conclusão}

O enfermeiro pediatra ao pautar o cuidado em evidências científicas necessita capacitar-se para utilizar os instrumentos que são os mais apropriados para a avaliação biopsicossociais das crianças nos vários cenários de atendimento à criança. Assim, recomenda-se a tradução, adaptação transcultural e validação do instrumento CD:H. Este estudo visa contribuir para o conhecimento dos enfermeiros pediatras no manejo da ansiedade da criança em unidades pediátricas e na proposição de intervenções possíveis no cuidado da criança, que diminuam seu sofrimento emocional durante a hospitalização.

\section{Referências}

1. Nóbrega RD, Collet N, Gomes IP, Holanda ER, Araújo YB. [Hospitalized school-age children: the meaning of a chronic condition]. Texto Contexto Enferm. 2010;19(3):425-33. Portuguese.

2. Santos PM, Silva LF, Depianti JR, Cursino EG, Ribeiro CA. Os cuidados de enfermagem na percepção da criança hospitalizada. Rev Bras Enferm. 2016;69(4):646-53.

3. Costa TS, Morais AC. A hospitalização infantil: vivência de crianças a partir de representações gráficas. Rev Enferm UFPE on line. 2017; 11(Supl. 1):358-67.

4. Silva JM. The drawing in the expression of feelings in hospitalized children]. Fractal: Rev Psicol. 2010;22(2):447-56. Portuguese.

5. Sadock BJ. Compêndio de Psiquiatria: ciências do comportamento e psiquiatria clínica. 9a ed. Porto Alegre: Art Med; 2007.

6. Bay EJ, Algase DL. Fear and anxiety: a simultaneous concept analysis. Nurs Diagn. 1999;10(3):103-11.

7. Gomes GL, da Nóbrega MM. Anxiety in children following hospitalization: a proposal for a nursing diagnosis. Rev Lat Am Enferm. 2015;23(5):963-70.

8. Mendes KD, Silveira RC, Galvao CM. Revisão integrativa: método de pesquisa para incorporação de evidências na saúde e na enfermagem. Texto Contexto Enferm. 2008;17(4):758-64.

9. Santos CM, Pimenta CA, Nobre MR. A estratégia pico para a construção da pergunta de pesquisa e busca de evidências. Rev Lat Am Enferm. 2007; 15(3):508-11

10. Galvão TF, Pansani TS, Harrad D. Principais itens para relatar revisões sistemáticas e meta-análises: a recomendação PRISMA. Epidemiol Serv Saude. 2015;24(2):335-42.

11. Castaneda A, McCandless BR, Palermo DS. The children's form of the manifest anxiety scale. Child Dev. 1956;27(3):317.

12. Reynolds CR, Richmond BO, What I. What I think and feel: a revised measure of children's manifest anxiety. J Abnorm Child Psychol. 1978;6(2):271-80. 
13. Reynolds CR, Richmond BO. Factor structure and construct validity of "what I think and feel": The Revised Children's Manifest Anxiety Scale. J Pers Assess. 1979;43(3):281-3.

14. Richmond BO, Millar GW, What I. Think and Feel: a cross-cultural study of anxiety in children. Psychol Sch. 1984;21(2):255-8.

15. Gorayeb MA, Gorayeb R. 0 que penso e sinto - Adaptação da Revised Childrens Manifest Anxiety Scale (RCMAS) para o português. Temas Psicol. 2008;16(1):1-11.

16. Birmaher B, Khetarpal S, Brent D, Cully M, Balach L, Kaufman J, et al. The Screen for Child Anxiety Related Emotional Disorders (SCARED): scale construction and psychometric characteristics. J Am Acad Child Adolesc Psychiatry. 1997;36(4):545-53.

17. Birmaher B, Brent DA, Chiappetta L, Bridge J, Monga S, Baugher M. Psychometric properties of the Screen for Child Anxiety Related Emotional Disorders (SCARED): a replication study. J Am Acad Child Adolesc Psychiatry. 1999;38(10):1230-6.

18. Isolan L, Salum GA, Osowski AT, Amaro E, Manfro GG. Psychometric properties of the Screen for Child Anxiety Related Emotional Disorders (SCARED) in Brazilian children and adolescents. J Anxiety Disord. 2011;25(5):741-8.

19. Potasz C, De Varela MJ, De Carvalho LC, Do Prado LF, Do Prado GF. Effect of play activities on hospitalized children's stress: a randomized clinical trial. Scand J Occup Ther. 2013;20(1):71-9.

20. Kovacs M. Children's depression inventory manual. Toronto: MultiHealth Systems; 1992

21. Beck AT, Ward CH, Mendelson M, Mock J, Erbaugh J. An inventory for measuring depression. Arch Gen Psychiatry. 1961;4(6):561-71.

22. Gouveia RV, Barbosa GA, Almeida HJ, Gaião AA. [nventário de Depressão Infantil - CDI: estudo de adaptação com Escolares de João Pessoa. J Bras Psiquiatr. 1995;44(7):345-9.

23. Golfeto JH, Veiga MH, Souza L, Barbeira C. Propriedade psicométricas do Inventário de Depressão Infantil (CDI) aplicado em uma amostra de escolares de Ribeirão Preto. Rev Psiquiatr Clin (Santiago). 2002;29(2):66-70.

24. Cruvinel M, Boruchovitch E, Santos AA. [Child Depression Inventory (CDI): analyses of its psychometrical properties]. Fractal: Rev Psicol. 2008;20(2):473-90. Portuguese.

25. Vianna RR. Avaliação dos níveis de ansiedade de uma amostra de escolares no Rio de Janeiro através da Escala Multidimensional de Ansiedade para Crianças (MASC-VB). Psicol Clin. 2009;21(2):493504.

26. March JS, Parker JD, Sullivan K, Stallings P, Conners CK. The Multidimensional Anxiety Scale for Children (MASC): factor structure, reliability, and validity. J Am Acad Child Adolesc Psychiatry. 1997;36(4):554-65.

27. March JS, Sullivan K, Parker J. Test-retest reliability of the Multidimensional Anxiety Scale for Children. J Anxiety Disord. 1999;13(4):349-58.

28. Nunes MM. Validade e confiabilidade da escala multidimensional de ansiedade para crianças [dissertação]. São Paulo: Universidade de São Paulo, Faculdade de Medicina; 2004.

29. Fioravanti AC, Santos LF, Maissonette S, Cruz AP, Landeira-Fernandez J. [Evaluation of the factorial structure of the trait anxiety scale of the STAI]. Aval Psicol. 2006;5(2):217-24. Portuguese.

30. Biaggio AM, Natalício L, Spielberger CD. Desenvolvimento da forma experimental em português do Inventário de Ansiedade Traço-Estado (IDATE), de Spielberger. Arq Bras Psic Apl. 1977;29(3):31-44.
31. La Greca AM, Dandes SK, Wick P, Shaw K, Stone WL. Development of the social anxiety scale for children: reliability and concurrent validity. J Clin Child Psychol. 1988;17(1):84-91.

32. La Greca AM, Stone WL. Social anxiety scale for children-revised: factor structure and concurrent validity. J Clin Child Psychol. 1993;22(1):17-27.

33. Li HC, Lopez V. Children's Emotional Manifestation Scale: development and testing. J Clin Nurs. 2005;14(2):223-9.

34. Park JH, Foster RL. Examining validity and reliability of the emotional reactions checklist with hospitalized children]. Pain Manag Nurs. 2015;16(4):579-86.

35. Kim JS, Park JH, Foster RL, Cheng S. Psychometric assessment of the Emotional Reactions Instrument-Korean (ERI-K) to measure Korean children's emotional reaction to hospitalization. J Transcult Nurs. 2011;22(1):31-9.

36. Kim JS, Park JH, Foster RL, Tavakoli A. Psychometric validation of Emotional Reaction Instrument-English to measure American children's emotional responses to hospitalisation. J Clin Nurs. 2014;23(11-12):1541-51.

37. Crandall M, Lammers C, Senders C, Savedra M, Braun JV. Initial validation of a numeric zero to ten scale to measure children's state anxiety. Anesth Analg. 2007;105(5):1250-3.

38. Tesler MD, Savedra MC, Holzemer WL, Wilkie DJ, Ward JA, Paul SM. The word-graphic rating scale as a measure of children's and adolescents' pain intensity. Res Nurs Health. 1991;14(5):361-71.

39. GiftAG. Visual analogue scales: measurement of subjective phenomena. Nurs Res. 1989;38(5):286-8.

40. Clatworthy S, Simon K, Tiedeman ME. Child drawing: hospital - an instrument designed to measure the emotional status of hospitalized school-age children. J Pediatr Nurs. 1999;14(1):1-9.

41. Clatworthy S, Simon K, Tiedeman M. Child drawing: hospital manual. J Pediatr Nurs. 1999;14(1):10-8.

42. Quintana AM, Arpini DM, Pereira CR, Santos MS. A vivência hospitalar no olhar da criança internada. Cienc Cuid Saúde. 2007;6(4):414-23.

43. Freitas PG. 0 desenho da figura humana e o desenho da pessoa doente na avaliação psicológica de crianças hospitalizadas [dissertação]. São Paulo: Instituto de Psicologia, Universidade de São Paulo, Instituto de Psicologia; 2008.

44. Dileo J. Interpreting children's drawings. New York: Brunner/ Mazel Publishers; 1983.

45. Koppitz E. Psychological evaluation of human figure drawings by middle school pupils. New York: Allyn and Bacon; 1984.

46. Dileo J. Young children and their drawings. New York: Brunner/ Mazel Publishers; 1970.

47. Skybo T, Ryan-Wenger N, Su YH. Human figure drawings as a measure of children's emotional status: critical review for practice. J Pediatr Nurs. 2007;22(1):15-28.

48. Buck JN. HTP: casa - árvore - pessoa, técnica projetiva do desenho: manual e guia de interpretação. 2a ed. São Paulo: Vetor; 2009.

49. Driessnack M. Children's drawings as facilitators of communication: a meta-analysis. J Pediatr Nurs. 2005;20(6):415-23.

50. Dolidze K, Smith EL, Tchanturia K. A clinical tool for evaluating emotional well-being: self-drawings of hospitalized children. J Pediatr Nurs. 2013;28(5):470-8.

51. Menezes M, Moré CL, Cruz RM. 0 desenho como instrumento de medida de processos psicológicos em crianças hospitalizadas. Aval Psicol. 2008;7(2):189-98. 